Revue des patrimoines

\title{
À la frontière entre la France et l'Espagne : la création d'une architecture nationale catalane. Étude des écrits du critique et historien d'art Raimon Casellas (1901-1905)
}

At the border between France and Spain: the creation of a Catalan national architecture. A study of the writings of the art critic and historian Raimon Casellas (1901-1905)

\section{Lucie Abdul-Lévêque}

\section{OpenEdition}

Édition électronique

URL : http://journals.openedition.org/insitu/20335

DOI : 10.4000/insitu.20335

ISSN : $1630-7305$

Éditeur

Ministère de la Culture

Référence électronique

Lucie Abdul-Lévêque, «À la frontière entre la France et l'Espagne : la création d'une architecture nationale catalane. Étude des écrits du critique et historien d'art Raimon Casellas (1901-1905) », In Situ [En ligne], 38 | 2019, mis en ligne le 15 février 2019, consulté le 10 décembre 2020. URL : http:// journals.openedition.org/insitu/20335; DOI : https://doi.org/10.4000/insitu.20335

Ce document a été généré automatiquement le 10 décembre 2020.

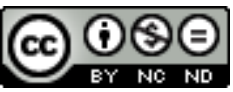

In Situ Revues des patrimoines est mis à disposition selon les termes de la licence Creative Commons Attribution - Pas d'Utilisation Commerciale - Pas de Modification 4.0 International. 


\section{À la frontière entre la France et l'Espagne : la création d'une architecture nationale catalane. Étude des écrits du critique et historien d'art Raimon Casellas (1901-1905)}

At the border between France and Spain: the creation of a Catalan national architecture. A study of the writings of the art critic and historian Raimon Casellas (1901-1905)

Lucie Abdul-Lévêque

1 Nous savons, depuis les ouvrages d'Anderson Benedict ${ }^{1}$, Éric Hobsbawm² ou Anne-Marie Thiesse $^{3}$, que les nations ne sont pas, comme il a été longtemps admis, des entités existant depuis des temps immémoriaux mais bien plutôt le fruit d'une construction entamée à la fin du XVIII ${ }^{\mathrm{e}}$ siècle. Comme le montre Michela Passini ${ }^{4}$, le rôle de l'art n'est pas anodin dans le processus de construction nationale. Les rapports qu'il entretient avec les gouvernements et les élites dévoués à la construction de la nation ${ }^{5}$ sont ambigus. Ainsi, les nationalismes influent sur la production artistique et vice versa ${ }^{6}$.

2 Cette situation d'une montée d'un nationalisme établissant des liens étroits avec l'art, et plus particulièrement l'architecture, se retrouve en Catalogne à travers le mouvement du modernisme, à la charnière entre les XIX et $\mathrm{XX}^{\mathrm{e}}$ siècles ${ }^{7}$. En 1901, le parti catalaniste de la Ligue régionaliste (Lliga Regionalista), fondé par Enric Prat de la Riba (1870-1917), remporte les élections législatives et municipales de Barcelone, marquant le triomphe du catalanisme. Premier parti catalaniste de masse, ses visions sont souverainistes ${ }^{8}$, puisqu'il considère que la Catalogne est une véritable nation qui doit aspirer au statut d'État ${ }^{9}$; il préfigure ainsi les partis catalanistes séparatistes du $\mathrm{XxI}^{\mathrm{e}}$ siècle $^{10}$. En tant que nation, la 
Catalogne apparaît comme un espace culturel dont les limites ne coïncident pas avec les frontières politiques du territoire espagnol ${ }^{11}$. Dans ce processus d'indépendance, l'architecture prend une grande place dans la mesure où elle devient l'un des critères essentiels à la définition et à l'affirmation d'une nation ${ }^{12}$. Pour compléter les études sur les rapports qu'entretiennent architecture et nationalisme en Catalogne à la fin du XIX ${ }^{e}$ siècle et au début $d u \mathrm{xx}^{\mathrm{e} 13}$, nous avons cherché à savoir comment le discours sur l'architecture participe à démontrer que la Catalogne est une région tout à fait différente du royaume espagnol, pouvant légitimement accéder au statut d'État-nation indépendant. La Catalogne entretenant par ailleurs des liens étroits avec son pays frontalier ${ }^{14}$, la France, État-nation que personne ne conteste, on peut se demander jusqu'à quel point l'Hexagone apparaît comme un modèle à suivre pour la Catalogne dans le domaine de la fabrication d'une architecture nationale.

3 Dans ces années de montée du catalanisme, une figure particulière et injustement méconnue ${ }^{15}$ se détache, celle de l'écrivain, journaliste, collectionneur, critique et historien d'art catalan Raimon Casellas (1855-1910). Il fréquente tout au long de sa vie le milieu culturel catalan (et plus particulièrement barcelonais) et rejoint en 1899 en tant que rédacteur en chef le journal du parti catalaniste cofondé par le politicien Prat de la Riba, La Veu de Catalunya (La Voix de Catalogne). Il est en contact permanent avec le reste de l'Europe, notamment la France, par des voyages, relations ou articles de journaux ${ }^{16}$. C'est en raison des liens étroits qui unissent Raimon Casellas à l'art, d'abord, au catalanisme, ensuite, et à la France, enfin, que cet intellectuel nous semble tout indiqué pour tenter de comprendre comment les discours sur l'architecture espagnole, française ou catalane, ont contribué à fabriquer une architecture nationale catalane spécifique, instrumentalisée pour servir un discours politique catalaniste. Notre étude, basée sur les écrits produits et conservés entre 1901 et $1905^{17}$ par Raimon Casellas, analyse l'influence de la France sur une architecture qui reflèterait une civilisation catalane particulière différente de l'architecture espagnole, et un caractère catalan unique, distinct du castillan.

\section{L'appartenance à une civilisation}

4 Le concept de "civilisation" est très lié à celui de «nation». Il désigne d'abord le progrès, le développement d'une société ${ }^{18}$. Au xix ${ }^{e}$ siècle, l'anthropologue britannique Edward Taylor le définit ainsi : «Le mot culture, ou civilisation, pris dans son sens ethnographique le plus étendu, désigne ce tout complexe comprenant à la fois les sciences, les croyances, les arts, la morale, les lois, les coutumes et les autres facultés et habitudes acquises par l'homme dans l'état social ${ }^{19}$ ». Ces concepts auxquels Taylor se réfère pour désigner la civilisation sont aussi ceux utilisés par Prat de la Riba pour caractériser une nation: progrès technique, religion, droit, coutumes et $\operatorname{arts}^{20}$. Ce lien entre civilisation, nation, art et, par extension, architecture, apparait clairement ici. Une société, pour se constituer en tant que nation, serait tenue à cette époque de posséder un art et une architecture sophistiqués, symboles de sa civilisation avancée. En outre, l'art national, et dans notre cas, l'architecture nationale, doit se distinguer des autres civilisations, des autres sociétés, des autres nations ${ }^{21}$.

5 La reconnaissance d'un peuple, pays ou région comme nation indépendante passant, à la fin du XIX ${ }^{e}$ siècle, par son appartenance à une civilisation, la Catalogne souhaite mettre en avant une civilisation qui lui est propre, bien distincte de celle de l'Espagne, basée sur une 
vision progressiste, une histoire et un art : trois notions qui trouvent leur résonance dans l'architecture. Dans ce processus de construction de civilisation catalane, la France joue un rôle primordial.

\section{Le rôle de la France dans l'affirmation d'une Catalogne progressiste face à une Espagne conservatrice}

6 La révolution industrielle qui touche la Catalogne tout au long du XIX ${ }^{e}$ siècle donne lieu à l'émergence d'une nouvelle classe sociale : la bourgeoisie ${ }^{22}$. Au même moment, l'Espagne pâtit, sur la scène européenne, d'une image de pays rétrograde, en retard sur le plan culturel par rapport aux pays nord-européens ${ }^{23}$. Le royaume souffre d'un complexe d'infériorité ${ }^{24}$ et éprouve des difficultés économiques et sociales ${ }^{25}$ qui le rendent incapable de répondre aux attentes de cette jeune bourgeoisie catalane ${ }^{26}$. Celle-ci, pour défendre ses intérêts, cherche à se détacher de l'Espagne pour se construire une nouvelle identité progressiste ${ }^{27}$, dont elle va trouver le modèle dans son pays frontalier : la France. Les jeunes artistes catalans, qui jugent alors leur culture démodée, anachronique, déphasée et provinciale, en somme, à l'image de celle de l'Espagne, aspirent à créer un art moderne et prennent également exemple sur la France, et plus particulièrement Paris, alors perçue comme la capitale de la modernitée ${ }^{28}$. C'est ainsi qu'en 1905, l'architecte catalan Josep Puig i Cadafalch (1867-1957) encourage les Barcelonais à faire les efforts nécessaires pour que leur ville devienne le «Paris $\mathrm{du}^{\mathrm{Midi}}{ }^{29}$ ».

7 Toutefois, pour Raimon Casellas et les catalanistes, la France représente bien plus qu'un modèle culturel permettant la construction d'une nouvelle identité catalane. Dans un article que le critique d'art rédige à l'occasion de la venue amicale d'une flotte de militaires français à Barcelone, on peut lire : «La France est pour nous le passage vers l'Europe, le chemin vers le monde, la route vers la civilisation. Ce qu'il convient n'est pas de rester sur place ni de nous isoler, comme une taupe dans son terrier, mais de vivre la vie moderne, la vie expansive, la vie qui communique avec le monde civilisé... ${ }^{30}$. La France n'est donc pas seulement vue comme un pays civilisé, sur la voie du progrès, qui ouvre la Catalogne à la modernité européenne. Elle est aussi un exemple indispensable dans la construction d'une civilisation catalane progressiste qui entend être reconnue sur la scène européenne.

8 Parallèlement, on observe en Catalogne comme dans le reste de l'Europe ${ }^{31}$, une redécouverte du passé médiéval. Les institutions encouragent le travail scientifique et les fouilles archéologiques ${ }^{32}$. Les références à la France sont présentes dans les discours de l'époque sur les monuments du Moyen Âge, dans la mesure où l'on y souligne la parenté entre les architectures française et catalane. Dans la revue française L'Art et l'Autel ${ }^{33}$, Georges Desdevises du Dézert explique en effet que le plan, les chapelles sombres et les étroites fenêtres de l'architecture catalane sont le fruit d'influences françaises méridionales :

Le gothique catalan est fils du gothique français du Midi. L'ampleur de la nef paraît avoir été l'idéal des maîtres méridionaux. Pour l'accentuer encore, l'architecte des Jacobins de Toulouse a divisé son vaisseau en deux galeries parallèles par une rangée de grosses colonnes, plantées dans l'axe même de l'église. Les Catalans ont divisé leur nef en trois allées par deux rangs de colonnes. Comme à Carcassonne, comme à Perpignan, les chapelles latérales sont restées basses et sombres, les hautes fenêtres de la claire voie ont été conservées, mais elles n'éclairent plus que les galeries latérales, et l'allée centrale, à peine plus élevée que les deux autres, ne 
prend jour que par d'étroites et basses fenêtres, ou par des roses minuscules, d'un effet un peu bizarre pour nos yeux.

Si l'on voit que les techniques architecturales françaises, et notamment la voûte d'ogives, ont eu un impact direct sur l'architecture religieuse catalane, il serait erroné de penser que les artistes catalans n'auraient fait que copier les nouveautés françaises. Ils s'en sont bien plutôt inspirés pour les adapter à leur propre région, créant ainsi leur propre style. Raimon Casellas écrit ainsi à propos des architectes catalans :

d'abord adopter, parmi les courants généraux, celui qui correspond le plus à son tempérament particulier, ensuite, assimiler la formule apprise, avec cette faculté innée de transformation que nous appliquons à tout ce qui ne vient pas de chez nous lorsque, alors que nous nous l'approprions, nous y imprégnons, des sortes d'essences, de germes et éléments de notre idiosyncrasie nationale. Un grand exemple de cela est la construction ogivale en Catalogne.

10 Cette technique originaire de France, les Catalans la «transform[ent] tout de suite si radicalement, la transfigur[ent] avec tant d'originalité, que selon Street, il n'y a pas d'école plus digne d'études que celle de l'architecture catalane des $\mathrm{XIV}^{\mathrm{e}}$ et XV $^{\mathrm{e}}$ siècles ${ }^{34}$ ». Georges Desdevises du Dézert précise quant à lui que «l'art catalan est en général très sobre » en matière d'architecture, et qu'«il réserve sa richesse pour les portails; ceux de la cathédrale de Lérida, de Barcelone, de Tarragone et de Valence en offrent un très bon modèle $»^{35}$.

11 On note un phénomène semblable avec les matériaux prédominants de l'architecture contemporaine catalane que sont, d'après Raimon Casellas, le fer et le verre ${ }^{36}$. Selon l'auteur, la manière de travailler ces matériaux a également été influencée par le nord de l'Europe. Mais de la même façon que les techniques de construction françaises ont été adaptées au territoire catalan, la manière de travailler ces matériaux en Catalogne n'a rien à voir avec celle que l'on peut trouver au-delà des Pyrénées. Elle est résistante, virile, robuste. Raimon Casellas écrit à ce titre :

Certes, il ne fait aucun doute que des faits historiques comme l'influence de l'art du nord aient contribué à ce bel aspect solide et vigoureux de notre fer historique. Tout cela est indubitable. On ne peut nier aucune de ces influences. Mais je crois plus ou moins qu'une chose essentielle a contribué à tout cela : le tempérament de notre race, confiant, concis, sobre, enclin à la robustesse plus qu'aux délicatesses. Ce que nous avons dit du fer peut être dit du verre, dans les arts industriels ${ }^{37}$.

Dans les textes de Raimon Casellas, ces matériaux et la technique qui les accompagne sont directement associés à un particularisme catalan: la virilité et la force de la "race » catalane. Ce terme de « race » est bien sûr ici à entendre dans son acception de l'époque, à savoir le classement de l'espèce humaine selon des critères morphologiques ou culturels, sans aucun fondement scientifique ${ }^{38}$. Ici, Raimon Casellas distingue le peuple catalan des autres peuples alentours (nous pensons bien évidemment à la France et à l'Espagne) plus que par un aspect physique, par un caractère (fort, viril, robuste) qui transparaît dans l'architecture. Son discours sur l'architecture catalane nourrit en réalité un discours nationaliste.

13 En résumé, pour défendre l'idée d'une Catalogne indépendante, Raimon Casellas s'appuie sur l'architecture pour inscrire la région dans une civilisation progressiste bien distincte de celle du reste de l'Espagne. Dans ses écrits, il met en avant une architecture moderne inspirée de la France, alors considérée comme étant à la pointe du renouveau, et ce, tant à l'époque médiévale que contemporaine. La frontière culturelle s'avère ainsi paradoxalement assez perméable avec la France et totalement hermétique avec l'Espagne, 
entité dont la Catalogne fait pourtant politiquement partie. C'est enfin pour légitimer l'affranchissement de la Catalogne de l'État espagnol que Raimon Casellas tente de démontrer qu'il existe bel et bien une civilisation catalane, représentée par une architecture unique qui n'est pas une pâle copie du style français mais bien une adaptation des innovations de ce dernier aux spécificités locales.

\section{L'architecture médiévale catalane comme porte-parole d'une histoire catalane spécifique}

14 Nous l'avons vu, pour être reconnue comme nation indépendante sur la scène européenne, la Catalogne se doit de faire partie d'une civilisation. Celle-ci ne se fonde pas seulement sur une vision progressiste, elle comporte également une culture propre, qui se constitue entre autres d'une histoire et d'un art. Au XIX siècle, tout peuple qui aspire au statut de nation promeut une histoire nationale individuelle, prétendument redécouverte, créée en vérité à partir de faits réels ou mythiques. Ces histoires nationales favorisent l'affirmation des peuples à l'échelle du continent européen, c'est pourquoi elles connaissent un tel développement ${ }^{39}$. La Catalogne ne fait pas exception et met elle aussi en avant une histoire nationale que reflètent les monuments. Dans cette région, c'est la période médiévale qui est remise à l'honneur, puisqu'elle renvoie à une époque où ce territoire faisait partie d'une vaste principauté souveraine et reconnue de tous, le royaume catalan-aragonais, riche et prospère, qui entretenait un rapport de force équilibré avec les deux grandes puissances qui le jouxtaient: le royaume de France au nord et les territoires d'Al-Andalous puis du royaume de Castille à l'est. Cette puissance trouve son apogée aux XIII ${ }^{\mathrm{e}}$ et $\mathrm{XIV}^{\mathrm{e}}$ siècles, alors que Catalogne et Aragon règnent sur la Méditerranée et occupent une large partie est de la péninsule Ibérique ${ }^{40}$. L'ère gothique est de ce fait considérée comme l'âge d'or par excellence de la Catalogne et bénéficie de l'image d'une époque idéalisée au cours de laquelle le peuple catalan était libre du joug castillan, dans un pays indépendant. C'est donc naturellement la fin du Moyen Âge catalan qui est utilisée pour fabriquer une histoire nationale catalane. Celle-ci prétend inscrire la région dans une civilisation et tisser un lien entre la période médiévale et contemporaine, afin de montrer que l'aspiration de la Catalogne à retrouver le statut de puissance indépendante vis-à-vis du royaume d'Espagne est légitime. En matière d'architecture, cette histoire est symbolisée par les monuments gothiques ${ }^{41}$.

Ainsi, ce sont par des références à la France, et non à l'Espagne, que la revalorisation d'une architecture médiévale catalane est justifiée. Cela transparaît dans un article de Raimon Casellas de mai 1904 où il répond aux détracteurs de l'art médiéval que ce n'est pas parce que d'illustres intellectuels de l'époque ont fustigé l'art de cette période qu'il ne vaut rien $^{42}$. Il ajoute d'ailleurs que tous les pays européens sont sur la voie de la valorisation de cette architecture, jusqu'aux États-Unis qui ne possèdent pourtant pas de tradition médiévale directe. Il avance enfin que si la France peut asseoir sa légitimité sur la scène internationale grâce à la revalorisation de son architecture gothique, la Catalogne devrait faire de même pour s'affirmer comme État-nation ${ }^{43}$.

Chez Raimon Casellas, on constate que le passé médiéval est très associé à la notion de civilisation développée plus haut. Mener des études sur l'art du Moyen Âge participe à nourrir l'histoire de la nation pour ancrer les territoires et leurs peuples dans une civilisation. On lit ainsi dans un article qu'il rédige à propos de l'exposition des Primitifs français : 
Mais rien n'est plus sûr, aujourd'hui, que le fait que l'actuelle revendication gothique ne soit pas une œuvre exclusivement française, mais bien un courant universel, une aspiration commune aux nations civilisées. Dû à un phénomène singulier de notre époque, les peuples les plus progressistes, les peuples qui vont au devant de la civilisation moderne, les peuples qui se sont révélés les plus aptes à notre époque à lutter pour la vie, que ce soit dans les domaines scientifique ou économique, politique ou social, sont clairement les peuples qui avec le plus d'amour et le plus de clairvoyance ont tourné leurs regards vers les arts des siècles du Moyen Âge, comme si, nostalgiques de cette époque, ils aspiraient à retrouver le chemin perdu ${ }^{44}$.

En revendiquant un art et une architecture gothiques, la Catalogne se définit alors comme une "nation civilisée », au même titre que sa voisine française et à la différence de l'Espagne, à laquelle Raimon Casellas ne fait même pas référence.

Dans ce même article, Raimon Casellas en appelle une nouvelle fois à la France pour expliquer que, plus une architecture tarde à être reconnue, plus cette reconnaissance est forte : «C'est pour cela que, dans un pays comme la France, où l'art ogival a été décrié en son sein pendant trois ou quatre siècles [...], la résurrection gothique des temps modernes a encore plus de signification qu'ailleurs ${ }^{45}$. » Il poursuit en faisant un parallèle direct avec la situation catalane : « Nous nous résignons en pensant que notre heure n'est pas arrivée, mais qu'un jour sans faute elle arrivera, de la même façon qu'en France ; même en France, il a fallu attendre plus d'un demi-siècle d'essais infructueux pour pouvoir toucher au triomphe d'aujourd'hui ${ }^{46}$.»

Concrètement, ce sont alors les cathédrales qui deviennent les symboles des nations légitimes ou en quête de légitimité. Elles apparaissent comme les plus à même de représenter une civilisation puisqu'elles sont à la fois le reflet d'un art et d'une histoire lointains. C'est le cas de l'Espagne avec le baroque churrigueresque, de la France avec la cathédrale de Chartres et de la Catalogne avec la Sagrada Familia.

L'existence d'une architecture castillane symbole de sa nation est démontrée par Georges Desdevises du Dézert. Dans l'article «L'art religieux en Espagne $»^{47}$, il écrit: "Ce qui donne aux cathédrales espagnoles un si puissant intérêt, c'est qu'elles sont restées telles qu'elles étaient au Moyen Âge, au temps où elles résumaient la vie religieuse et intellectuelle de la nation ». Ici, la nation espagnole est clairement associée à ses cathédrales. L'auteur affirme ensuite que l'Espagne possède une architecture nationale, le baroque churrigueresque (fig. 1), qualifié de « souvent affreux » et de « marque nationale de l'État espagnol $»^{48}$. Or, si l'art d'un pays est effectivement le reflet de sa civilisation, on imagine facilement quelle image le lecteur se fait de l'Espagne : une nation, certes, mais incapable de produire un bel art. Ceci est symptomatique de la vision que la France a de l'Espagne à cette époque : une "contrée arriérée ${ }^{49}$ ", un pays lointain, exotique, marqué par la misère, la violence, le brigandage et la mendicité ${ }^{50}$. 
Figure 1

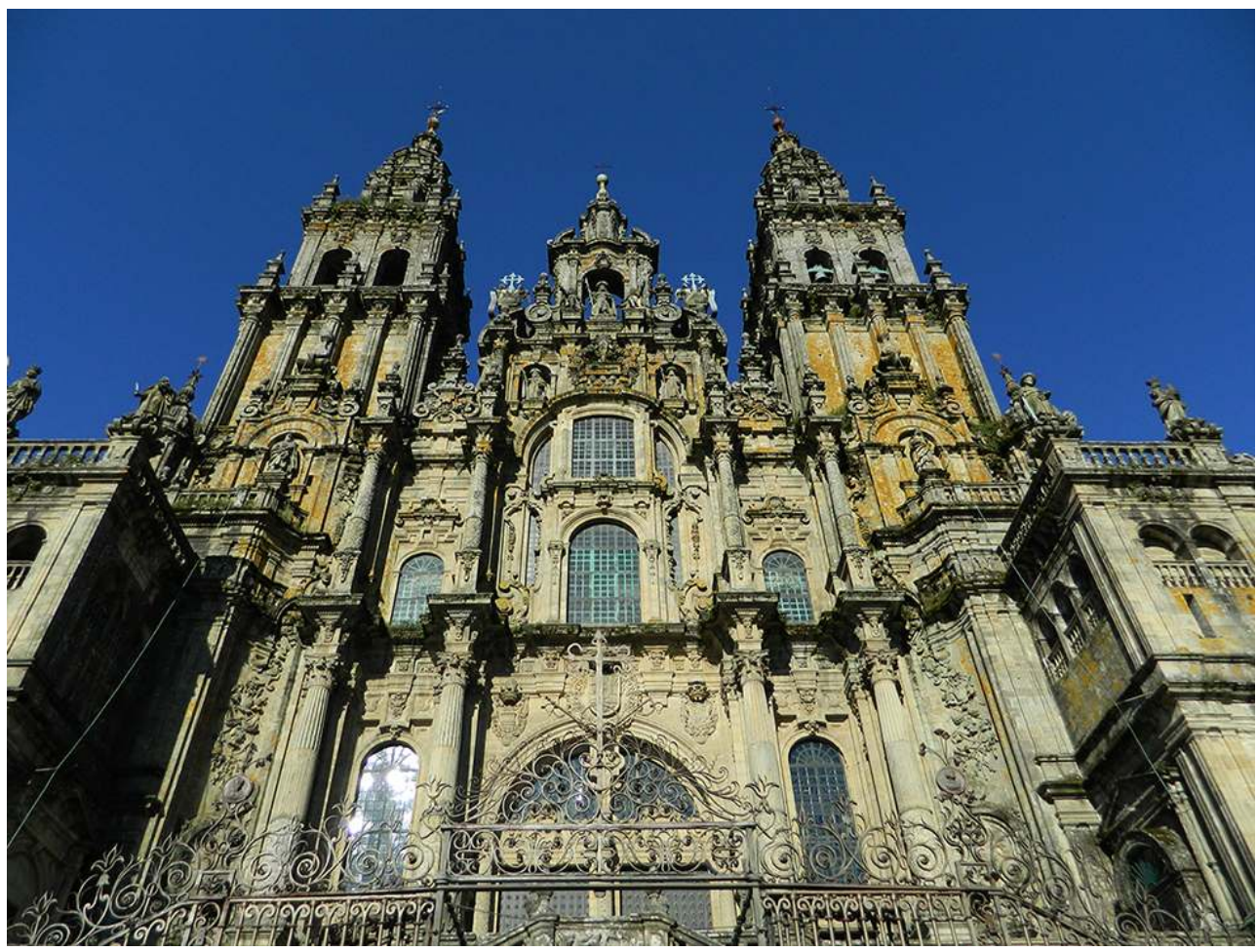

Exemple de façade de style baroque churrigueresque. Façade dite d'« Obradoiro » de la cathédrale de Saint-Jacques de Compostelle, en Espagne, 1750

Phot. Buymistr Andriy. 2012. (C) Buymistr Andriy.

21 Pour se détacher de cette image négative de l'Espagne, la Catalogne soutient également posséder une architecture nationale qui lui est propre. Celle-ci est symbolisée par la Sagrada Familia (fig. 2) et son architecte Antoni Gaudí (1852-1926). 
Figure 2

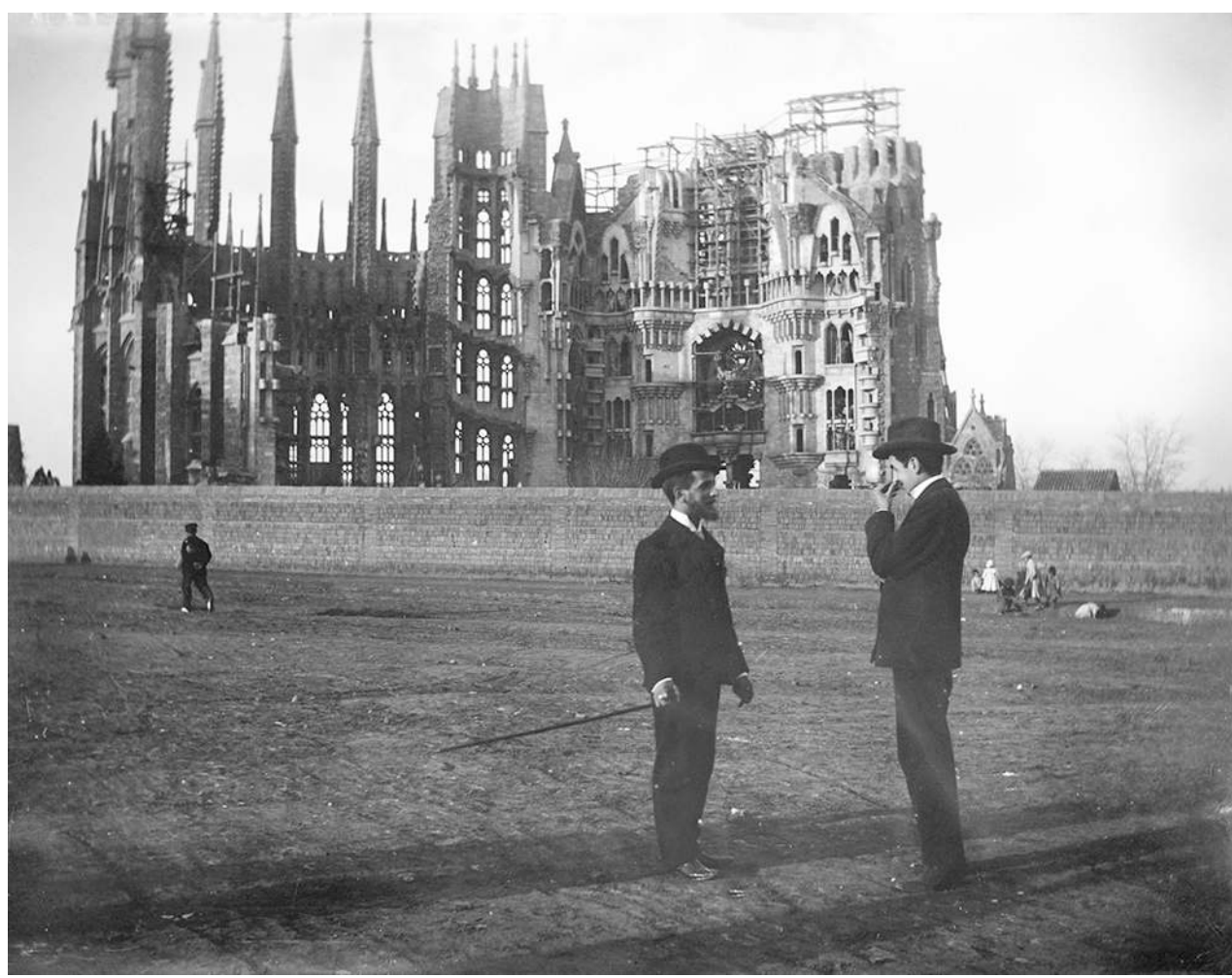

La Sagrada Familia, 1905. Reproduction moderne du négatif original en verre.

Phot. Gili i Roig Baldomer. Museu d'Art Jaume Morera, 2013. (c) Museu d'Art Jaume Morera.

Dans une lettre adressée à Raimon Casellas, l'architecte moderniste Bonaventura Conill i Montobbio (1876-1946) définit ce dernier comme « un homme qui est prophète en sa patrie » et souligne à propos de sa cathédrale « que l'on ne voit cela que chez nous $»^{51}$. Il montre ainsi bien la spécificité de la Catalogne par rapport à ses voisins. À la différence des discours sur le Moyen Âge, la région ne cherche plus seulement à se distinguer de l'Espagne mais aussi de la France. On assiste alors à la création d'une nouvelle frontière culturelle, cette fois-ci, contemporaine. Le fait que Bonaventura Conill i Montobbio aille jusqu'à encourager la reconnaissance de la Sagrada Familia comme monument national ${ }^{52}$ traduit également cette émancipation catalane. Ce discours est partagé par des auteurs français comme Georges Desdevises du Dézert. Il considère lui aussi cette cathédrale comme représentante de l'architecture catalane lorsqu'il écrit :

M. Gaudí élève, avec la foi et la patience d'un grand bâtisseur du Moyen Âge, son fantastique sanctuaire de la Sagrada Familia. À l'extrémité du Passeo de San-Joan [...], se dessine, dans une large enceinte, la haute et fière silhouette de l'immense église en construction qui, une fois achevée, sera la merveille de Barcelone ${ }^{53}$.

Gaudí est comparé à un "bâtisseur du Moyen Âge", ce qui permet de l'inscrire directement dans la lignée des architectes médiévaux. Par là, il incarne avec son monument la continuité de l'histoire et de la civilisation catalanes. La Sagrada Familia et son architecte se posent ainsi comme symboles de l'architecture nationale catalane, non seulement aux yeux des Catalans, mais aussi aux yeux d'étrangers comme Georges Desdevises du Dézert. 

d'une architecture nationale française. Le sculpteur Rodin compare ainsi la cathédrale de Chartres, cette "œuvre maitresse " érigée en monument de l'art national, avec l'Acropole, reconnue comme symbole de l'architecture grecque et de sa civilisation: «Chartres, notre cathédrale, splendide entre toutes! N'est-ce pas l'Acropole de la France $?^{54} »$. Ceci vaut en général pour toutes les grandes cathédrales de France. En effet, Émile Mâle ${ }^{55}$ fait le parallèle entre le Parthénon grec et la cathédrale de Reims pour justifier le fait que la France, comme la Grèce, possède sa propre architecture nationale mais aussi un « génie » français, d'égale importance au « génie " grec ${ }^{56}$.

L'idée selon laquelle un artiste moderne doit se projeter dans l'avenir tout en gardant des racines ancrées dans une tradition ancienne est très développée en cette fin du XIXe, comme nous le montrent les articles des critiques ou journalistes André Beaunier ${ }^{57}$, Henry Crosnier de Varigny ${ }^{58}$ ou Georges Desdevises du Dézert. Ce dernier explique qu'avant de créer, les hommes ont toujours imité leurs semblables et ce qui existait déjà. En se référant à la Sagrada Familia, il met en avant un art moderne catalan original qui garde toutefois un lien avec sa culture gothique :

Après avoir copié, ils ont voulu créer à leur tour [...]. L'église sera de style catalan, avec trois nefs, de hauteur presque égale, séparées par de hautes colonnes monocylindriques [...]. Le dessin général [de la façade] est gothique : trois porches s'évasant de dedans en dehors, et couronnés de trois pignons aigus, mais ce gothique donne l'impression de l'irréel et de l'invraisemblance, jamais vu, jamais rêvé ${ }^{59}$.

Raimon Casellas insiste lui aussi sur le fait que l'art moderne catalan doit s'inscrire dans une tradition régionale ${ }^{60}$. Il écrit par exemple : « Dès lors, nous pourrions dire que l'heure est venue d'avoir un art propre, un art fondé sur le goût et sur la tradition de notre race ${ }^{61}$ ». Avec ce terme de "race ", nous retrouvons ici l'idée chère à Casellas que le peuple catalan est bel et bien différent des autres, porteur d'une culture ("goût ») et d'une histoire («tradition») uniques qui lui seraient propres et se refléteraient dans l'art. Son propos est que l'art et l'architecture modernes catalans doivent prendre racine dans l'histoire régionale, au même titre que l'art moderne des autres grandes nations, parmi lesquelles la France.

Ainsi, c'est bien sûr le style gothique qui inspire les architectes catalans. Nous l'avons déjà vu avec le cas de la Sagrada Familia, mais le Monument au deuxième mystère glorieux du Rosaire $^{62}$ (fig. 3), de l'architecte catalan Bonaventura Bassegoda (1862-1940) et du sculpteur catalan Josep Reynés i Amigó (1850-1926), peut également être cité. Pour Raimon Casellas, sa partie centrale pourrait être celle d'un retable gothique ${ }^{63}$. Enfin, à propos de la maison-atelier du peintre Santiago Rusiñol, où des éléments d'origine médiévale cohabitent avec un style à la fois moderniste et néogothique ${ }^{64}$, le critique d'art souligne les références à l'architecture du Moyen Âge et une continuité certaine dans l'architecture catalane médiévale et moderne ${ }^{65}$. Ces exemples illustrent bien la particularité à ses yeux des architectes modernes catalans qui, s'ils s'inspirent de l'art ancien, savent toujours apposer sur leurs œuvres une touche de nouveauté et d'originalité. 
Figure 3

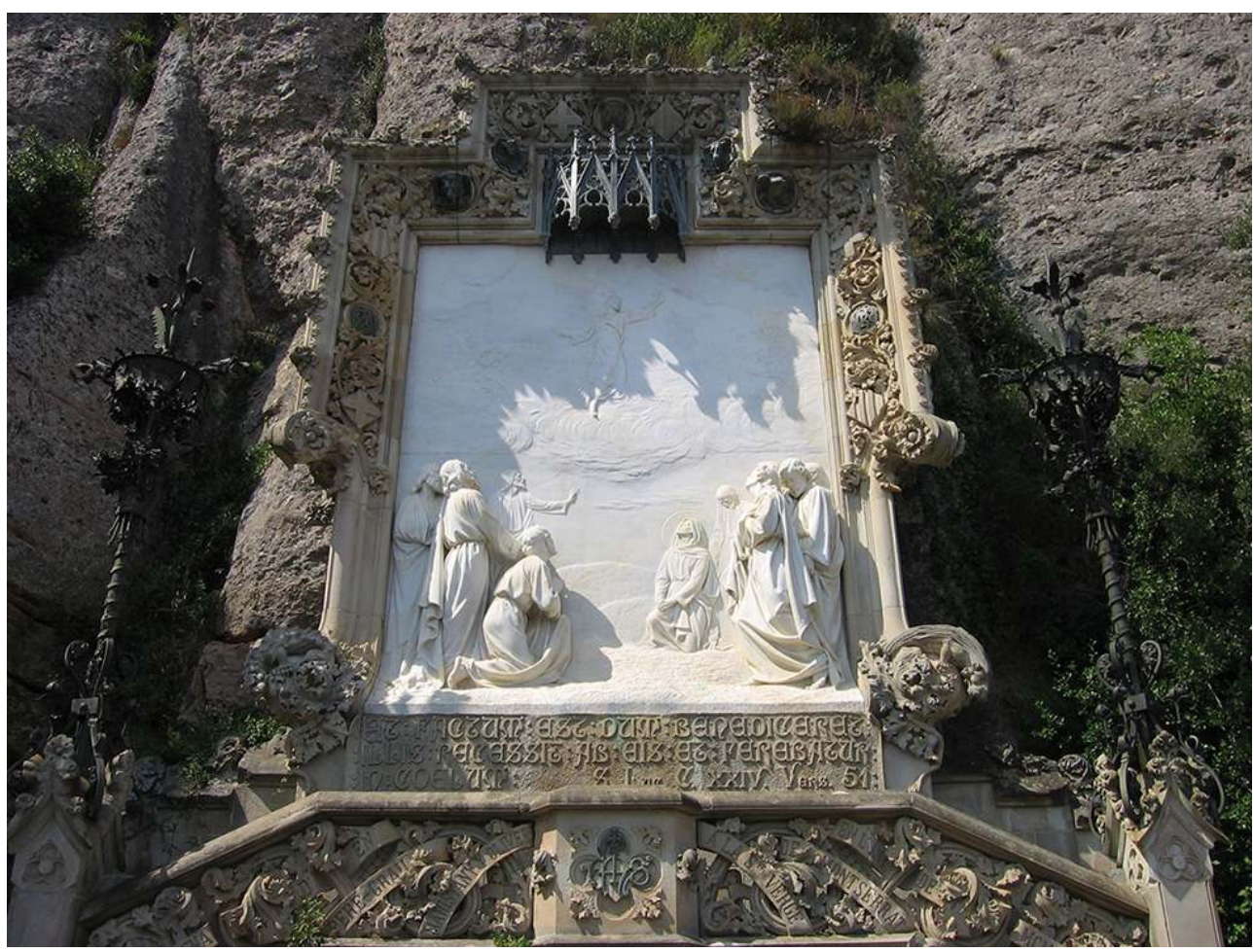

Bonaventura Bassegoda et Reynés i Amigo Josep, monument au deuxième mystère glorieux du Rosaire monumental de Montserrat, 1903.

Phot. Canaan, 2012. (c) Canaan.

Pour faire partie d'une civilisation et ainsi, être reconnue comme nation indépendante, la Catalogne crée une histoire nationale dont l'architecture médiévale est l'emblème essentiel, car elle renvoie à un glorieux passé catalan. En plus d'inscrire la Catalogne dans une histoire riche car lointaine et de minimiser le poids de l'histoire castillane et, par là même, du royaume de Castille, la redécouverte du passé médiéval catalan offre la possibilité de rapprocher la région catalane des grands États-nations progressistes qui sont eux aussi sur la voix de la mise en valeur de leur art gothique, comme la France. Paradoxalement, l'étude du corpus souligne que cette mise en valeur du Moyen Âge catalan s'effectue par des références à la France plutôt qu'à l'Espagne. Les cathédrales, parce qu'elles sont le reflet d'un art et d'une histoire lointaine, deviennent les symboles des nations légitimes ou en quête de légitimité. Pour finir, l'architecture moderne catalane puise quant à elle son inspiration directement dans les monuments médiévaux de la région, appliquant ainsi les principes de théoriciens français comme Viollet-le-Duc ${ }^{66}$. En créant une continuité entre le passé et le présent, elle se pose comme véritable porteparole de l'histoire catalane en démontrant que la Catalogne est toujours aussi puissante qu'au Moyen Âge, et en offrant à son peuple une identité à laquelle il puisse se référer, qui n'est ni espagnole ni française. 


\section{L'architecture comme représentante d'un caractère catalan distinct du castillan}

\section{Le reflet architectural des personnalités catalanes et castillanes}

Dans les discours catalanistes du tournant du XIX et $\mathrm{XX}^{\mathrm{e}}$ siècle, les monuments ne servent pas seulement à mettre en avant une histoire et une culture particulières, ils traduisent également le caractère même du peuple catalan, qui apparaît comme bien distinct du castillan.

Pour Raimon Casellas, la personnalité du peuple catalan, tant à son époque ${ }^{67}$ qu'à l'époque médiévale, transparaît dans l'architecture, et plus particulièrement, dans ses matériaux que sont le fer et le verre ${ }^{68}$, considérés comme "l'âme même de la race » catalane ${ }^{69}$. Le caractère catalan, associé au fer et au verre, est décrit en termes plutôt positifs. Selon Raimon Casellas, la verrerie catalane est franche, virile et «masculine, par sa vigueur gracieuse » et « son aspérité exquise $»^{70}$, tandis que le fer est fort et « sincère ${ }^{71}$. Paré de toutes les qualités, le caractère catalan ressort alors comme authentique, résistant, courageux et puissant. En revanche, le caractère espagnol est présenté comme très imparfait, à l'image de son peuple. C'est ce qu'allègue Georges Desdevises du Dézert lorsqu'il écrit : «L'art espagnol est comme [l'Espagne], incomplet et violent, capricieux et inégal ${ }^{72}$. »

Dans les discours de Raimon Casellas et de Georges Desdevises du Dézert, l'architecture est clairement instrumentalisée pour servir des propos catalanistes qui définissent un caractère catalan, bien différent du caractère espagnol, et déprécient ce dernier.

\section{Une France laïque, une Catalogne chrétienne, ou l'appropriation de la chrétienté par la Catalogne}

2 De par sa situation géographique, il n'est pas aisé pour la Catalogne de déterminer un caractère catalan, et plus largement, une civilisation catalane, indépendamment des deux grandes puissances, France et Espagne, entre lesquelles elle se trouve prise en étau. Si le caractère catalan est ainsi défini comme bien distinct de l'espagnol, on peut se demander s'il est pour autant semblable au français. Les discours de ce tout début $\mathrm{du} \mathrm{xx}^{\mathrm{e}}$ siècle sur l'architecture nous éclairent sur ce point, en ce sens qu'ils attribuent à cette dernière le rôle de représentante de la spiritualité des deux peuples, français et catalan.

En France, les discours sur l'architecture montrent que la laïcité, ou plutôt, l'absence de croyances, est une manière de définir le caractère français. C'est du moins l'opinion de l'historien de l'art Henri Bouchot, pour qui l'une des caractéristiques de l'art français, et donc de son peuple, est la laïcité ${ }^{73}$, entendue à l'époque comme le caractère de ce qui n'est ni ecclésiastique, ni religieux ${ }^{74}$. Pour lui, les artistes du Moyen Âge ne sont pas mus par une foi religieuse, mais plutôt comparables aux travailleurs qui lui sont contemporains. Les peintres, comme les ouvriers, refléteraient le contexte socio-politique d'une époque donnée. Sa vision de l'art est donc sociale et laïque ${ }^{75}$. Ces opinions reflètent les écrits de Viollet-Le-Duc pour qui « les cathédrales s'élevaient bien plus comme des édifices affectés à une destination civile qu'à un service religieux ${ }^{76}$ ". Cette indépendance vis-à-vis des conceptions religieuses est considérée par ces différents auteurs comme une spécificité 
majeure du caractère français. Ils appliquent cette mentalité moderne aux artistes contemporains mais aussi, curieusement et sans réel fondement, aux artistes médiévaux. De cette façon, ils confèrent au caractère laïque une dimension intemporelle qui légitime encore plus ce trait dans la construction identitaire française. À l'époque cependant, la position d'Henri Bouchot ou de Viollet-Le-Duc sur le caractère laïque des artistes français médiévaux est loin d'être partagée. Pour Émile Mâle, au contraire, l'art sacré ne peut être conçu sans foi ${ }^{{ }^{7}}$. Louis Dimier, fervent catholique, ne pouvait pas imaginer l'idée d'un art laïque ${ }^{78}$. Il est intéressant de noter que cette controverse au sujet des artistes, inspirés ou non par leur foi, s'inscrit dans un contexte particulier en France, celui du large débat sur la laïcité et le Concordat, qui aboutit en 1905 à la loi de séparation des Églises et de l'État ${ }^{79}$ et contribue à faire de la neutralité en matière de religion l'un des caractères principaux de l'identité française.

Pour s'affirmer comme nation, la Catalogne s'approprie le christianisme. Elle se détache en cela de la France tout en se rapprochant de la très catholique Espagne. L'un des meilleurs exemples pour illustrer ce propos est sans doute celui de la Vierge de Montserrat.

Figure 4

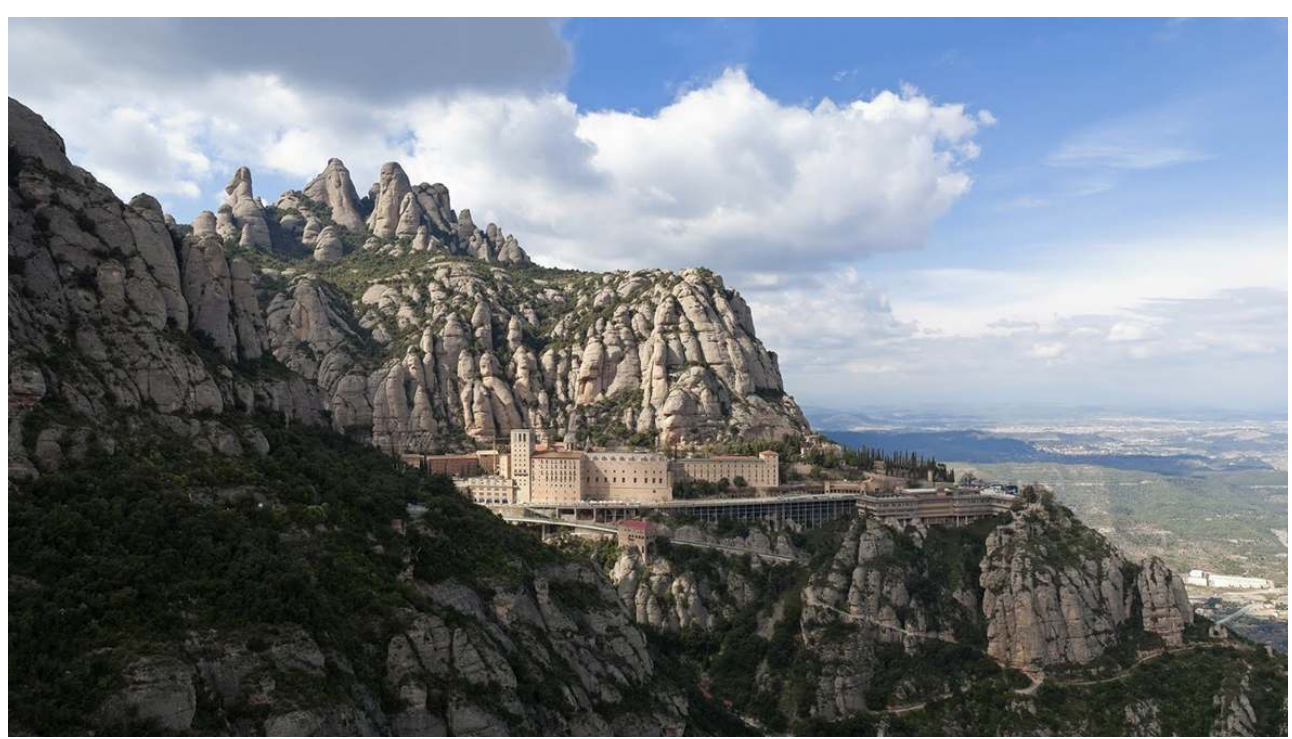

Le monastère de Montserrat, dans la montagne du même nom, en Catalogne.

Phot. Ene, Alexandru, 2011. (c) Alexandru Ene. 
Figure 5

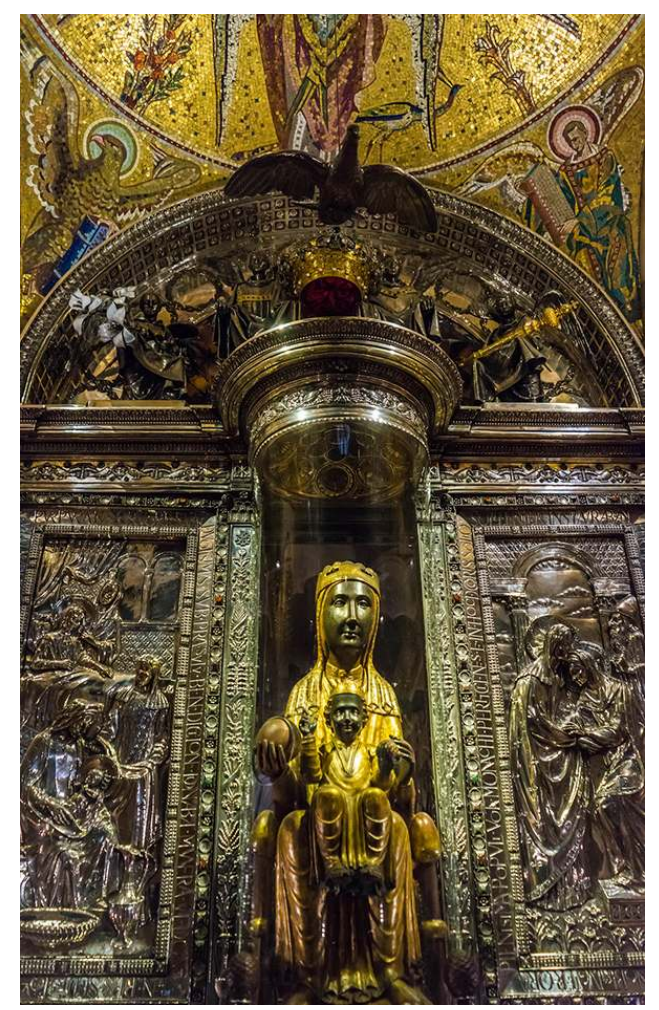

Statue de la Vierge de Montserrat, XII siècle.

Phot. Maria, Michelle. 2016. @ Michelle Maria.

Montserrat, qui est à la fois une montagne, un monastère et le nom de la Vierge qui y est vénérée (fig. 4, fig.5), donne une dimension à la fois géographique, physique et symbolique au catalanisme, permettant ainsi la matérialisation de la nation catalane ${ }^{80}$. $\mathrm{Au}$ sein d'une nation en construction, la religion, ou plus précisément, les icônes religieuses, apparaissent comme une force de cohésion ${ }^{81}$. La Vierge de Montserrat fait l'objet d'un culte en Catalogne depuis les temps médiévaux mais ce n'est qu'à partir de la fin du XIX siècle qu'elle prend une signification catalaniste à proprement parler et qu'elle devient un emblème de la région ${ }^{82}$. Elle est considérée, ainsi que l'écrit Raimon Casellas, comme la " mère de tous les Catalans ${ }^{83}$ ", et donc, par extension, la mère de la nation. Créer une dimension filiale entre le peuple et son symbole donne à ses individus un sentiment d'appartenance à une même communauté, ce qui développe parallèlement le sentiment patriotique. Si l'on ajoute à cela que la Vierge est honorée depuis un millénaire, voire davantage $e^{84}$, l'idée que le peuple catalan fait partie d'une nation ayant toujours existé est d'autant plus forte.

Les manifestations de vénération de cette Vierge au début $\mathrm{du} \mathrm{xx}^{\mathrm{e}}$ siècle sont particulièrement perceptibles lors de l'inauguration du Monument au deuxième mystère glorieux du Rosaire (voir fig. 3), au cours des fêtes du monastère, dans l'été 1903. La cérémonie y est décrite comme " aussi intime et aussi catalane que dévote dans tous ses aspects $^{85}$ ", et la Vierge est clairement associée à la nation catalane. Raimon Casellas rapporte en effet que, sur les supports du monument « splendidement décorés avec deux monstres inspirés des types de gargouilles les plus beaux de chez nous, apparaissent les blasons de la Catalogne et de sant Jordi [saint Georges, patron de la Catalogne] ainsi que 
les symboles des quatre évangélistes en bronze ${ }^{86}$ ». À la fin de l'article, l'auteur apporte par ailleurs son soutien et ses félicitations au mécène Pere Guerau Maristany i Oliver ${ }^{87}$ qui « a honoré notre estimée Patronne, a honoré l'art de la terre, de notre Catalogne désirée, s'honorant lui-même ${ }^{88}$ ".

Ce thème d'une Catalogne chrétienne traverse les frontières, relayé par des auteurs français qui le voient transparaître dans l'architecture. Selon Georges Desdevises du Dézert ${ }^{89}$, l'un des principaux traits de la Barcelone de ce début $\mathrm{du} \mathrm{xx}^{\mathrm{e}}$ siècle est son caractère religieux. Il écrit en effet : «Barcelone devient un centre artistique de premier ordre, et l'art religieux s'y montre merveilleusement vivant. » Il prend en outre l'exemple des calvaires, des pierres tombales ou des chapelles funéraires de Puig y Cadafalch (fig. 6) construits pour le cimetière ornemental de la ville. Il s'attarde aussi sur la Sagrada Familia d'Antoni Gaudí qui l'élève avec « foi ». Avec ces articles étrangers, il apparaît que le caractère chrétien catalan n'est pas seulement admis par son propre peuple mais également par son voisin français, ce qui peut apparaître comme le début d'une reconnaissance de l'identité catalane sur la scène européenne.

Figure 6

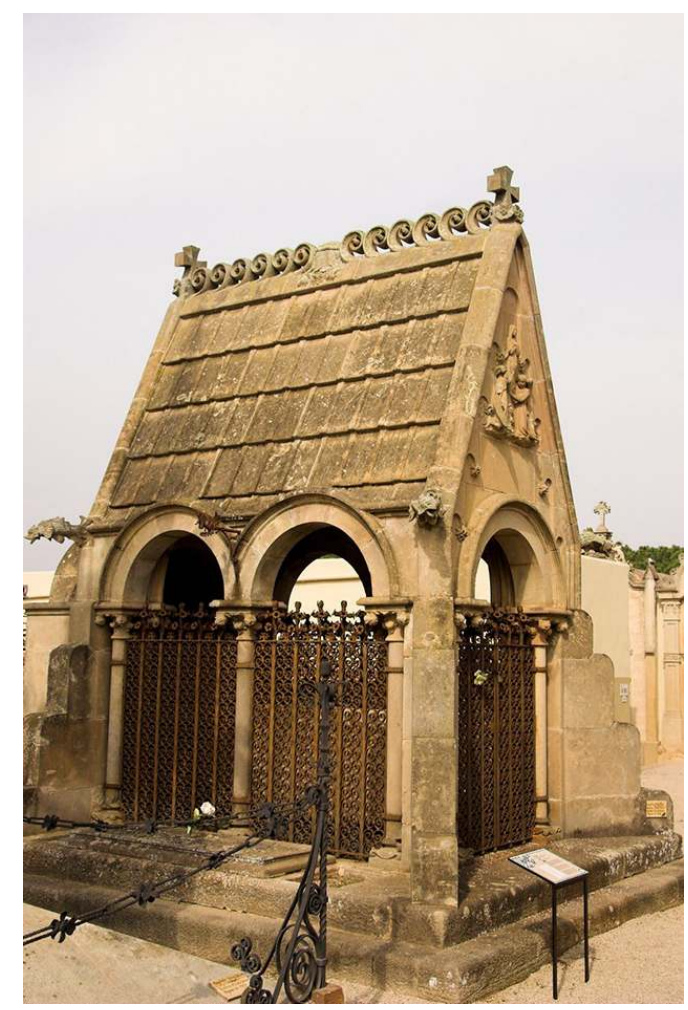

Josep Puig i Cadafalch, monument funéraire à la famille Costa i Macià dans le cimetière de Lloret del Mar de Catalogne, 1902.

Phot. Amadalvarez, 2008. (c) Amadalvarez.

Il faut, enfin, remarquer que lorsque les auteurs français ou catalans font référence à cette foi chrétienne comme l'une des principales caractéristiques du peuple catalan, aucune allusion n'est faite à l'Espagne. C'est comme si cet État-nation pourtant très catholique se voyait dépossédé de l'un de ses principaux attributs, au profit d'une Catalogne qui paraît s'approprier cette religion de manière à mieux peser face au royaume castillan. 


\section{Conclusions}

Les discours des premières années du $\mathrm{Xx}^{\mathrm{e}}$ siècle sur l'architecture cités dans cette étude, qu'ils soient français, espagnols ou plutôt catalans, véhiculent l'idée que la Catalogne est une région tout à fait différente du reste de l'Espagne, qui peut légitimement accéder au statut d'État-nation indépendant. L'analyse des articles de Raimon Casellas sur l'architecture révèle qu'il existe une identité culturelle catalane indépendante des frontières politiques. Celles-ci ne sont d'ailleurs pas très bien définies pour les catalanistes de l'époque ${ }^{90}$. Si le territoire catalan est assez semblable à celui que nous connaissons aujourd'hui (fig. 7), celui de la nation catalane va au-delà des délimitations régionales, englobant les espaces où la langue catalane est parlée, parmi lesquels le Roussillon français ${ }^{91}$.

\section{Figure 7}

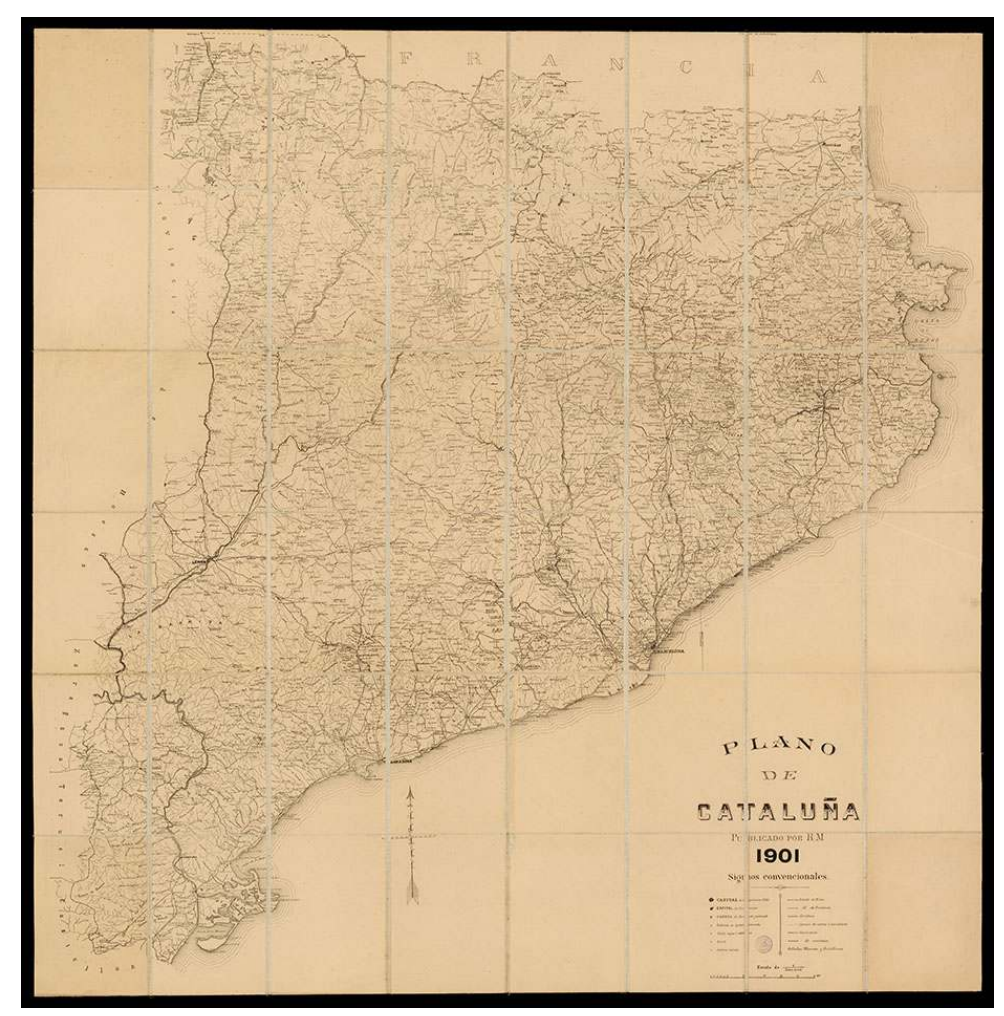

Ramon Morera, carte de Catalogne, 1901. Repro. Institut Cartogràfic i Geològic de Catalunya, 15 octobre 2012. Registre : RM.182802.

(c) Institut Cartogràfic i Geològic de Catalunya.

Même si l'architecture catalane s'est construite en réaction à ses deux pays limitrophes, l'Espagne, à qui elle s'oppose, la France, dont elle s'inspire dans une certaine mesure, elle est tout à fait spécifique. La fabrication d'une architecture nationale catalane est, paradoxalement, bien plus influencée par son pays frontalier, la France, que par l'Espagne, pays dont la région fait pourtant partie. L'impact des discours français sur les discours catalans est en effet indéniable, contribuant à l'émergence d'une esthétique architecturale catalane moderne particulière. Indirectement, ces discours français participent donc à fabriquer une architecture nationale catalane dont le but est de servir 
le message catalaniste en faveur de l'indépendance de la Catalogne de l'État espagnol. Malgré ce que laisse croire le discours catalaniste, l'identité catalane ne se constitue donc pas uniquement par elle-même, bien au contraire. Peut-on alors réellement parler d'une architecture nationale catalane, et plus généralement, d'un art et d'une identité culturelle catalans dans la mesure où ils ne sont pas le fruit unique du peuple catalan? Si l'on considère que les identités sont toujours le produit d'une construction qui s'établit par rapport aux autres, on peut alors penser qu'en effet, il existerait une identité catalane, et a fortiori, une architecture nationale catalane qui se seraient forgées en réaction à l'Espagne et à la France.

\section{NOTES}

1. - BENEDICT, Anderson. L'Imaginaire national. Réflexions sur l'origine et l'essor du nationalisme [1983]. Paris : La Découverte, 1996.

2. - HOBSBAWM, Eric. Nations et nationalisme depuis 1780 : programme, mythe, réalité [1990]. Paris : Gallimard, 1997.

3. - THIESSE, Anne-Marie. La Création des identités nationales: Europe XVIII ${ }^{e}-\mathrm{XX}{ }^{e}$ siècle. Paris : Seuil, 1999.

4. - PASSINI, Michela. La Fabrique de l'art national : le nationalisme et les origines de l'histoire de l'art en France et en Allemagne, 1870-1933. Paris : Éd. de la Maison des Sciences de l'homme, 2012. Voir aussi : LAUGÉE, Thierry, RABILLER, Carole (dir.). Critique d'art et nationalisme. Regards français sur l'art européen au XIX ${ }^{e}$ siècle. Bruxelles : Peter Lang, 2017.

5. - GELLNER, Ernest. Nations et nationalisme [1983]. Paris : Payot, 1989, p. 72-73.

6. - Voir par exemple MARFANY, Joan-Lluís. «Modernismo Catalan y fin de siglo europeo. Algunas reflexiones ». Dans GARCIA i ESPUCHE, Albert. El Modernisme. Cat. exp., Barcelone, Museu d'Art Modern, Parc de la Ciutadella, 10 octobre 1990-13 janvier 1991. Barcelone: Olimpiada Cultural/Lunwerg, 1990, vol. 1, p. 36-39.

7. - Voir par exemple ROHRER, Judith C. « Modernisme i neogotic en l'arquitectura ». Dans ibid., p. 323-324.

8. - MICHONNEAU, Stéphane. «Espagne ». Dans GUILLAUME, André, LESCURE, Jean-Claude et MICHONNEAU, Stéphane. L'Europe, des nationalismes aux nations. Paris : Sedes, 1996, p. 371-373, 384.

9. - Selon Prat de la Riba, l'Espagne doit devenir un «État composé » de plusieurs États-nations politiquement indépendants (voir ibid). On entend par le terme de « Nation » une « communauté humaine dans laquelle les individus se sentent unis par des liens à la fois matériels et spirituels » et dont les membres « ressentent que cette communauté est différente des autres nations »; par celui d'"État ", une "société politique résultant de la fixation, sur un territoire délimité par des frontières, d'un groupe humain présentant des caractères plus ou moins marqués d'homogénéité culturelle et régie par un pouvoir institutionnalisé»; et par celui de "Nationalisme », la recherche de la légitimité politique de la Nation qui « exige que les limites ethniques coöncident avec les limites politiques et de préférence au sein d'un État ». Pour « Nation », voir la préface d'ANDRIEUX, Jean-Yves, CHEVALLIER, Fabienne et KERVANTO NEVANLINNA, Anja. Dans Idée nationale et architecture en Europe, 1860-1919: Finlande, Hongrie, Roumanie, Catalogne. Rennes : PUR, 2006 ; pour « État », voir la définition du Dictionnaire Larousse [en ligne] http://www.larousse.fr/ 
dictionnaires/francais/\%C3\%89tat/31318?q=\%C3\%A9tat\#31250 [consulté le 23/08/2015]; pour " Nationalisme », voir GELLNER, Ernest. Nations et nationalisme. Op. cit., p. 12.

10. - Voir la préface de CAPDEVILA, Joaquim. Dans LLADONOSA LATORRE, Mariona. La Construcció de la catalanitat: Evolució de la concepció d'identitat nacional a Catalunya, 1860-1990. Lleida : Edicions de la Universitat de Lleida, 2013, p. 12.

11. - Sur les notions d'« espace» et de "territoire », voir LAFONT, Robert. "Permanence des espaces et transitivité des territoires: entre Loire, Maseta et Arc méditerranéen ». Dans SERRANO, Carlos et ZIMMERMANN, Marie-Claire. Le Discours sur la nation en Catalogne aux XIX ${ }^{e}$ et XX ${ }^{e}$ siècles. Hommage à Antoni M. Badia i Margarit. Paris : Éditions hispaniques, 1996, p. 87.

12. - KERVANTO NEVANLINNA, Anja. "The role of the arts in the construction of national identities». Dans ANDRIEUX, Jean-Yves, CHEVALLIER, Fabienne et KERVANTO NEVANLINNA, Anja. Idée nationale et architecture en Europe... Op. cit.

13. - Certaines études ont déjà été menées, comme celles de Mireia Freixa et Fabienne Chevallier dans ibid.

14. - AUBERT, Paul. « La France et la Catalogne : une histoire culturelle, 1888-1937 ». Dans LÉAL, Brigitte et OCANA, Maria Teresa. Paris Barcelone: de Gaudí à Miró. Cat. exp., Paris, Galeries nationales du Grand Palais, 9 octobre 2001-14 janvier 2002, Barcelone. Museu Picasso, 28 février-26 mai 2002. Paris : Réunion des musées nationaux, 2011, p. 9 et p. 55-65.

15. - La seule étude scientifique portant sur Raimon Casellas est la thèse de Jordi Castellanos, qui a été publiée : CASTELLANOS, Jordi. Raimon Casellas i el modernisme. Barcelone : Curial/Abadia de Montserrat, 1983.

16. - Raimon Casellas se rend à Paris lors de l'Exposition universelle de 1889. Dans les années 1890, il est également très proche des peintres Ramon Casas et Santiago Rusiñol qui exercent régulièrement à Paris. Enfin, le fonds Raimon Casellas conservé à la Biblioteca nacional de Catalunya (BNC) regorge d'articles de journaux français lui ayant appartenu.

17. - Le corpus se base sur des écrits produits et conservés par Raimon Casellas compris entre 1901 et 1905. Il comporte d'abord des articles de journaux écrits par Raimon Casellas et publiés dans La Veu de Catalunya entre 1901 et 1904, tous consultables sur le site de l'ARCA (Arxiu de Revistes Catalanes Antigues), qui les a numérisés : http://www.bnc.cat/digital/arca/index.php [consulté le 31/01/2019]. Il comprend ensuite des notes et brouillons rédigés par Raimon Casellas rassemblés dans le fonds Raimon Casellas de la Biblioteca nacional de Catalunya et enfin, des articles de journaux français et des correspondances lui ayant appartenu retrouvés dans ce même fonds (NB : même s'il s'agit d'articles publiés dans des périodiques largement diffusés, nous avons choisi de donner leur cote dans ce fonds). Dans cet article, toutes les citations provenant de documents en catalan ou en espagnol ont été traduites en français par nos soins.

18. - KAUFMANN, Pierre. «CULTURE - Culture et civilisation» Encyclopcedia Universalis [en ligne] : http://www.universalis-edu.com.acces-distant.bnu.fr/encyclopedie/culture-culture-et-

civilisation/ [consulté le 18/08/2015].

19. - CAZENEUVE, Jean. "CIVILISATION» Encyclopcedia Universalis [en ligne]: http:// www.universalis-edu.com.acces-distant.bnu.fr/encyclopedie/civilisation/ [consulté le 18/08/2015].

20. - Enric Prat de la Riba, dans son ouvrage La Nacionalitat catalana, paru en 1906, considère la nation comme une "entité naturelle, avec d'une part une histoire, une culture, une langue propres, sortes de produits naturels de la terre, et d'autre part, un art et un droit, c'est-à-dire un esprit unique et caractéristique d'une vie collective. » Voir MICHONNEAU, Stéphane. « Espagne ». Art. cit, p. 372.

21. - C'est ce que nous indique la définition du Larousse au mot «culture »: " Ensemble des phénomènes matériels et idéologiques qui caractérisent un groupe ethnique ou une nation, une civilisation, par opposition à un autre groupe ou à une autre nation ». «culture » Dictionnaire 
Larousse [en ligne], http://www.larousse.fr/dictionnaires/francais/culture/21072? q=culture\#20950 [consulté le 18/08/2015].

22. - SUDRIÀ, Carles. « Modernité d'une capitale industrielle ». Dans SANCHEZ, Alejandro (dir.). Barcelone 1888-1929: modernistes, anarchistes, noucentistes ou la création fiévreuse d'une nation catalane. Paris : Autrement, 1992, p. 37-39.

23. - RIQUER I PERMANYER, Borja de. «El Modernisme, una aventura cultural ». Dans RIQUER i PERMANYER, Borja de, CABANA i VANCELL, Francesc, DOMENECH i GIRBAU, Lluísi... [et al.]. Modernisme i modernistes. Barcelone : Lunwerg Editores, 2001, p. 11.

24. - Notamment depuis la perte de ses colonies. Voir : AUBERT, Paul. «L'influence idéologique et la politique de la France en Espagne de la fin du XIX ${ }^{\mathrm{e}}$ siècle à la Première Guerre mondiale (1875-1918) ». Dans ÉTIENVRE, Jean-Pierre et URQUIJO GOITIA, José Ramon de. España, Francia y la Comunidad europea. Actas del segundo Coloquio hispano-francés de historia contemporánea celebrado en Aix-en-Provence [y Marseille] los días 16, 17 y 18 de junio de 1986. Madrid: Casa de Velázquez, 1989, p. 58-59; TÉMIME, Émile. «France et Espagne dans la seconde moitié du XIX ${ }^{\mathrm{e}}$ siècle: le déclin d'une influence ». Dans ibid., p. 248-249; GIARD, Noémie. "La difficile reconnaissance française d'une architecture "catalane" ". Dans ANDRIEUX, Jean-Yves, CHEVALLIER, Fabienne et KERVANTO NEVANLINNA, Anja. Idée nationale et architecture en Europe... Op. cit. p. 195.

25. - MICHONNEAU, Stéphane. « Espagne ». Art. cit., p. 248-249.

26. - SANCHEZ, Alejandro. " Manchester espagnol, rose de feu, Paris du Sud... ». Dans SANCHEZ, Alejandro (dir.). Barcelone 1888-1929... Op. cit., p. 15-17.

27. - CASASSAS, Jordi. « Bataille et ambiguïtés du catalanisme ». Dans ibid., p. 129.

28. - CASACUBERTA, Margarida. "Portrait de la littérature catalane avec Paris en arrière-plan ». Dans LÉAL, Brigitte, OCANA, Maria Teresa. Paris Barcelone : de Gaudí à Miró... Op. cit., p. 27.

29. - LAHUERTA, Juan José. « À propos de l'architecture et la ville ». Dans ibid., p. 79.

30. - CASELLAS, Raimon. « Al dia - Domassos al balcó ». La Veu de Catalunya, 14 juillet 1899, p. 1.

31. - Id. «A propósit de l'Exposició dels Primitius Francesos ». La Veu de Catalunya, 25 mai 1904, p. 1.

32. - GUITART, Josep. "The Rediscovery of Ancient Barcelona ». Dans RAEBURN, Michael (dir.). Homage to Barcelona. The city and its art, 1888-1936. Cat. exp., Londres. Hayward Gallery, nov. 1985 fév. 1986. Londres: Thames and Hudson, 1986, p. 111-113. Fabienne Chevallier nous apprend aussi que, outre des vestiges romains, de nombreux édifices religieux des $\mathrm{X}^{\mathrm{e}}$ et $\mathrm{XII}^{\mathrm{e}}$ siècles sont mis au jour, comme les monastères de Ripoll ou de Poblet. Cet intérêt pour l'histoire de la Catalogne s'était déjà fait sentir dans les années 1870 , avec la constitution de musées comme le « Museu Provincial d'Antiquitats» installé dans la chapelle Sainte-Agathe (alors ancienne chapelle du palais des Rois d'Aragon) à Barcelone, ou la création du musée d'Histoire en 1888 (centré sur l'histoire de la Catalogne et de Barcelone) alors situé dans le jardin de la Ciutadella, réuni en 1902 avec le musée des Reproductions artistiques pour former le musée des Arts décoratifs et d'Archéologie (introduction de Fabienne CHEVALLIER à la partie sur « La Catalogne : Renaissance et puissance d'une nation ». Dans ANDRIEUX, Jean-Yves, CHEVALLIER, Fabienne, KERVANTO NEVANLINNA, Anja. Idée nationale et architecture en Europe... Op. cit., p. 163-167).

33. - DESDEVISES du DÉZERT, Georges. «L'art religieux en Espagne ». L'Art et l'Autel. Revue de beauté chrétienne, janvier 1903, p. 338-332 (BNC, Manuscrits, fonds Raimon Casellas [Bloc 8/4] Ms. 5244/40-9).

34. - CASELLAS, Raimon. «Cosas d'Art - Exposició d'Art Antich - Pintura gótica catalana. III ». La Veu de Catalunya, 23 octobre 1902, p. 3.

35. - DESDEVISES du DÉZERT, Georges. «L'art religieux en Espagne ». Art, cit.

36. - « Le fer et le verre : voyez là les deux industries d'art préférées de la Catalogne historique » ( CASELLAS, Raimon. «Ferros d'Art - Colecció de 200 láminas representant treballs de forja, dibuixadas per en Lluís Labarta ». La Veu de Catalunya, 11 juillet 1901, p. 1). 
37. - Ibid, p. 1.

38. - « race ». Dictionnaire Larousse [en ligne] : http://www.larousse.fr/dictionnaires/francais/ race/65899?q=race\#65153 [consulté le 24/07/2016].

39. - Dans la préface des actes du colloque Idée nationale et architecture en Europe, les auteurs écrivent d'ailleurs : «La fin du $\mathrm{XIX}^{\mathrm{e}}$ siècle est ainsi un objet d'étude exigeant pour l'histoire complexe du continent européen : tout se passe alors comme si les nations avaient convoqué leur riche passé - historique, mythologique ou imaginaire - pour mieux affirmer leur personnalité et en somme, pour exister dans l'Europe de ce temps" (ANDRIEUX, Jean-Yves, CHEVALLIER, Fabienne, KERVANTO NEVANLINNA, Anja. Idée nationale et architecture en Europe. Op. cit., p. 8).

40. - Le royaume catalan-aragonais occupe alors l'actuel Aragon, l'actuelle Catalogne mais aussi la région de Valence, de Murcie, les îles d'Ibiza, de Majorque, de la Sicile, de la Sardaigne et même, à la fin du XIV ${ }^{\mathrm{e}}$ siècle, les villes de Constantinople et d'Athènes (MORERA, Jean-Claude. Histoire de la Catalogne, au delà et en deça des Pyrénées. Paris : L'Harmattan, 1992, p. 36 et p. 48-59; ZIMMERMANN, Michel et Marie-Claire. Histoire de la Catalogne. Paris : PUF, 1997, p. 18-29).

41. - L'architecte Bonaventura Bassegoda i Amigo écrit que c'est grâce à l'« état politico-social de la Catalogne qui jouissait de la plénitude de sa condition nationale " qu'un art médiéval catalan riche et digne d'intérêt a pu éclore (BASSEGODA, Bonaventura. «Exposición de arte antiguo. II. Pintura ». Diario de Barcelona, 13 octobre 1902, p. 12076-12078 (BNC, Manuscrits, fonds Raimon Casellas [Bloc 6/3] Ms. 5244/14-14).

42. - Ces détracteurs se réclament des grands penseurs ayant dénigré l'art médiéval pour justifier leur position, entre autres de Voltaire, pour qui «les prestigieuses statues du XIII ${ }^{\mathrm{e}}$ siècle [...] faisaient l'effet d'enfants ridicules", ou Soufflot, qui «menaçait d'accoutrer ses vieilleries de grandes voûtes au milieu de la façade de Notre-Dame» (CASELLAS, Raimon. "A propósit de l'Exposició dels Primitius Francesos ». Art. cit., p. 1).

43. - «Les États-Unis regroupent des copies de millions de monuments gothiques de la vieille Europe, et remplissent les halls de leurs musées de reproductions de frontons et de claustres ogivaux, que l'on a fait venir du Trocadéro, et emploient le critique André Michel pour qu'il aille, comme il est allé, à Washington et à Montréal, à Boston et à Chicago, donner des séries de conférences publiques expliquant l'œuvre de la cathédrale de Chartres, de Notre-Dame de Paris, de Bourges, Amiens, Reims, Dijon et Rouen... » (Ibid.).

44. - Ibid.

45. - Ibid.

46. - Ibid.

47. - DESDEVISES du DÉZERT, Georges. « L'art religieux en Espagne ». Art. cit.

48. - «Le churrigérisme [sic] ne compte guère aujourd'hui que des détracteurs, et il faut bien avouer qu'il est souvent affreux. Mais, mal pour mal, il est encore préférable au pauvre style du dix-septième siècle. Quand elle est servie par une main habile, son étonnante fantaisie est parfois gracieuse et donne bien cette impression de somptuosité théâtrale, qui est comme la marque nationale de l'État espagnol. » (Ibid.).

49. - GIARD, Noémie. "La difficile reconnaissance française d'une architecture "catalane" ». Art. cit., p. 195.

50. - TÉMIME, Émile. «France et Espagne dans la seconde moitié du XIX siècle : le déclin d'une influence ». Art. cit., p. 248-249.

51. - CONILL, Bonaventura. Correspondance adressée à Raimon Casellas depuis Vilobí, datée du 17 novembre 1905 (17 f.) (BNC, Manuscrits, fonds Raimon Casellas [Bloc 1/1] Ms. 5229/3-1).

52. - «Si Gaudí a renoncé à toutes les incomparables propositions qui lui ont été offertes pour partir à l'étranger, c'est parce qu'il a cru en sa patrie [...]. De la même manière que l'on déclare implicitement monument national celui du Dr. Robert, on devrait faire une déclaration claire et catégorique similaire pour le temple de la Sagrada Familia " (Ibid.).

53. - DESDEVISES du DÉZERT, Georges. « L'art religieux en Espagne ». Art. cit. 
54. - RODIN, Auguste. "Chartres ». (1f.). Dans Les Cathédrales de France. Paris : Armand Colin, 1914, chap. XII, p. 111-112 (BNC, Manuscrits, fonds Raimon Casellas [Bloc 8/13] Ms. 5244/49-31).

55. - Émile Mâle était connu en Catalogne, et plus particulièrement de Raimon Casellas, qui lisait la revue de La Gazette des beaux-arts, dans laquelle écrivait l'historien de l'art. Institut d'Estudis Catalans. Anuari, 1909-1910. Barcelone : IEC, 1911, p. 733 ; SOLER, Josep, CASTAÑO i GARCIA, Joan et VILA, Josep. « Els Misteris i l'art gòtic ». La Rella. nº 19 [document électronique]. Institut d'Estudis Comarcals del Baix Vanalopó, 2006, p. 139: http://www.raco.cat/index.php/Rella/article/ view/72249/125395 [consulté le 03/11/2017].

56. - PASSINI, Michela. La Fabrique de l'art national... Op. cit., p. 221.

57. - « [Face au changement, les conservateurs] invoquent des traditions, mais lesquelles ? Toute l'histoire est pleine de renoncements à des traditions, ou plutôt, la tradition n'est qu'une transmission continue du passé au présent, qui prend ce qu'il veut ou ce qu'il peut, corrige, retranche, ajoute. Une tradition qui prétend s'arrêter à un moment donné n'est plus une tradition, c'est une borne. Retourner en arrière ? Mais pour habiter où ? [...] Nous ne parlons donc pas de retourner aux maisons détruites, mais plutôt tâchons de bâtir des maisons plus belles » (BEAUNIER, André. «Les Précurseurs». Le Figaro, 24 mai 1904 (BNC, Manuscrits, fonds Raimon Casellas [Bloc 6/5] Ms. 5244/16-8). Voir sur le site: https://gallica.bnf.fr/ark:/12148/ bpt6k2866228/f1 [consulté le 31/01/2019].

58. - Il explique que la société est le fruit de la collaboration entre les novateurs, les inventeurs et les imitateurs, les détenteurs de la tradition : « [L'œuvre] résulte de la collaboration de novateurs divers et nombreux, les derniers ayant utilisé l'œuvre des premiers » (VARIGNY, Henri CROSNIER de. «Les bases de la sociologie, d'après Tarde ». Le Temps, 20 juillet 1904, Causerie scientifique, La nature et la vie (1f.) (BNC, Manuscrits, fonds Raimon Casellas [Bloc 8/5] Ms. 5244/41-12). Voir sur le site : https://gallica.bnf.fr/ark:/12148/bpt6k237941k/f3 [consulté le 31/01/2019].

59. - DESDEVISES du DÉZERT, Georges. « L'art religieux en Espagne ». Art. cit.

60. - « De la même manière que l'influence des époques passées [...] s'est toujours fait sentir dans l'évolution éternelle des arts, si nous devons suivre une tradition, il n'y a pas besoin d'aller chercher en dehors de chez nous, nous l'avons bien assez présente dans notre patrimoine national » (CASELLAS, Raimon. « Cosas d'Art - Concurs de copias de la Exposició de Art Antich ». La Veu de Catalunya, 15 janvier 1903, p. 1).

61. - Ibid.

62. - Ce monument, inauguré en 1903, fait partie du Rosaire monumental de Montserrat, un ensemble de monuments et sculptures religieux situés dans le massif de Montserrat en Catalogne, à proximité du monastère du même nom.

63. - « Le style dominant est le gothique de l'époque catalane, riche et sobre à la fois, d'un dessin assez ferme pour rendre compte de la patte de Bassegoda, si elle ne se faisait pas déjà sentir » ( CASELLAS, Raimon. «Desde Montserrat - Inauguració del segón Misteri de Gloria del Rosari Monumental ». La Veu de Catalunya, 28 et 29 juillet 1903, p. 2).

64. - Parmi les éléments d'origine médiévale présents dans cette maison-atelier, on distingue entre autres des baies vitrées provenant de l'ancien château féodal de Sitges reconverti en maison consistoriale, ou un font baptismal $\mathrm{du} \mathrm{Xv}^{\mathrm{e}}$ siècle issu du sanctuaire de la Vierge du Vinyet (« Museo del Cau Ferrat. El edificio». Museus de Sitges [en ligne] : http://museusdesitges.cat/es/ museo/cau-ferrat/el-edificio [consulté le 14/11/2018].

65. - «Dans un lieu comme celui-ci, on comprend si intimement l'œuvre de nos forgerons médiévaux, créée grâce à des moyens scientifiques et de l'inspiration, de l'assurance technique et une liberté artistique » (CASELLAS, Raimon. «Ferros d'Art - Colecció de 200 láminas representant treballs de forja, dibuixadas per en Lluís Labarta ». Art. cit., p. 1).

66. - Les édifices construits par l'architecte catalan Lluís Domènech i Montaner (1850-1923) en sont un très bon exemple. Voir à ce titre LAHUERTA, Juan José. « À propos de l'architecture et la ville ». Art. cit., p. 86. 
67. - À l'époque du modernisme, les éléments industriels prennent une place prépondérante dans l'architecture. Ils sont mis en valeur et on ne cherche plus à les dissimuler. Le fer, la terre cuite, l'émail ou la brique font leur apparition sur les façades des édifices. Les architectes s'efforcent de souligner la structure même de leurs constructions en laissant apparaître les matériaux structurels comme le fer ou la brique. Voir DOMENÈNEC i GIRBAU, Lluís. «Arquitectes modernistes ». Dans Modernisme i modernistes. Op. cit., p. 35-45.

68. - « Le fer et le verre : voyez là les deux industries d'art préférées de la Catalogne historique » ( CASELLAS, Raimon. «Ferros d'Art - Colecció de 200 láminas representant treballs de forja, dibuixadas per en Lluís Labarta ». Art cit., p. 1).

69. - Raimon Casellas écrit à propos du fer et du verre : «Le plus fort et le plus faible, le plus opaque et le plus transparent, le plus dur et le plus fragile [...] ont été les matériaux que valorisèrent nos artistes du passé pour y imprégner leur idéal individuel de beauté, avec quelque chose de très énergique et courageux, qui est l'âme même de la race » (ibid.).

70. - «Dans la verrerie catalane, il y a une franchise et une virilité qui n'échappent pas à l'examen affectueux et arrêté. Ainsi, de la même manière que nous pourrions dire de la verrerie vénitienne, si délicate, volatile et minutieuse, qu'elle est féminine, de la verrerie catalane il se pourrait dire qu'elle est masculine, par sa vigueur gracieuse, par son aspérité exquise » (ibid.).

71. - « Tout y est sincère, tout y est force, tout y est virilité. Chaque coup de maillet, clairement visible, est comme un cri de la matière brute, faite esclave par la volonté et l'idéal de l'homme créateur [...]. La liberté d'une technique sommaire et d'une intention première, jointe à cet esprit de force, de virilité que nous avons commenté au début, constituent les caractères principaux de la ferronnerie catalane » (ibid.).

72. - DESDEVISES du DÉZERT, Georges. «L'art religieux en Espagne ». Art. cit.

73. - «Cette prééminence du laïque sur le prêtre est une des caractéristiques de notre vieil art français » (BOUCHOT, Henri, DELISLE, Léopold, GUIFFREY, Jules. Exposition des primitifs français : au Palais du Louvre (Pavillon de Marsan) et à la Bibliothèque Nationale. Paris: Palais du Louvre et Bibliothèque nationale, 1904, p. 32).

74. - AUGÉ, Claude et LAROUSSE, Pierre. Nouveau Larousse illustré: dictionnaire universel encyclopédique. Paris: Larousse, 1898, tome 5, p. 538 : https://archive.org/details/ nouveaularoussei05laro/page/n7 [consulté le 31/01/2019].

75. - Il écrit d'ailleurs que dans le futur, «l'histoire de la classe ouvrière laïque prendra [...] une importance particulière. Et ce sont les œuvres de ces artisans oubliés que le heureux hasard de nos curiosités et de nos snobismes mettra tout à coup au premier rang des études rétrospectives " (ibid.).

76. - VIOLLET-LE-DUC, Eugène. Histoire d'un hôtel de ville et d'une cathédrale. Paris : J. Hetzel, 1878, p. 115. Cité par MARTIN, François-René. « Henri Bouchot et la seconde redécouverte des maîtres anciens ». Dans THIÉBAUT, Dominique, MARTIN, François-René et LORENTZ, Philippe. Primitifs français. Découvertes et redécouvertes. Cat. exp., Paris, musée du Louvre, 27 février-17 mai 2004. Paris : Réunion des musées nationaux, 2004, p. 32.

77. - Dans son premier grand livre, L'Art religieux du XIII siècle en France, dans lequel il étudie la "pensée de l'Église s'exprimant dans l'art », il proteste vivement contre l'interprétation laïque de l'art gothique. En 1892, il affirme en outre dans un article sur les chapiteaux romans du musée des Augustins (Toulouse) qu' « il n'y a qu'un moyen d'expliquer le Moyen Âge, c'est d'entrer aussi profondément que possible dans la pensée chrétienne" (MÂLE, Émile. "L'enseignement de l'histoire de l'art dans l'Université ». Revue universitaire, t. I, 1894, p. 10-20, cité par PASSINI, Michela. La Fabrique de l'art national... Op. cit., p. 154).

78. - MARTIN, François-René. «La Gloire des primitifs français (1904-1945)». Dans THIÉBAUT, Dominique, MARTIN, François-René et LORENTZ, Philippe. Primitifs français... Op.cit., p. 62 ; PASSINI, Michela. «DIMIER, Louis». INHA [en ligne]. 3 novembre 2008: http://www.inha.fr/ spip.php?article2288 [consulté le 7/10/2012]. 
79. - C'est ce que souligne François-René Martin dans « Henri Bouchot et la seconde redécouverte des maîtres anciens ». Art. cit., p. 32.

80. - SERRANO, Carlos. «La réinvention de Montserrat : une vierge très ancienne pour une nation nouvelle ». Dans SERRANO, Carlos et ZIMMERMANN, Marie-Claire. Le Discours sur la nation en Catalogne aux XIX ${ }^{e}$ et XX siècles. Op. cit., p. 416.

81. - HOBSBAWM, Eric. Nations et nationalisme depuis 1780... Op. cit., p. 92-93.

82. - SERRANO, Carlos. « La réinvention de Montserrat... ». Art. cit., p. 405-417.

83. - CASELLAS, Raimon. « Desde Montserrat - Inauguració del segón Misteri de Gloria del Rosari Monumental ». Art. cit., p. 2.

84. - SERRANO, Carlos. " La réinvention de Montserrat... ». Art. cit., p. 416.

85. - CASELLAS, Raimon. « Cosas d'Art - Dos quadros den Limona ». La Veu de Catalunya, 18 juillet 1902, p. 1.

86. - Id. « Desde Montserrat - Inauguració del segón Misteri de Gloria del Rosari Monumental ». Art. cit., p. 2.

87. - La famille Maristany était une puissante famille de la région de Montserrat.

88. - Ibid.

89. - DESDEVISES du DÉZERT, Georges. « L'art religieux en Espagne ». Art. cit.

90. - Raimon Casellas revient sur la nécessité de définir les limites du territoire catalan de manière concrète dans un brouillon d'article sur la « forme physique de la Catalogne ». Il observe que cela fait quelques années que l'on assiste à l'expansion de la culture intellectuelle et des revendications politiques catalanes, phénomènes par ailleurs très positifs selon lui, mais que personne ne sait réellement définir les limites du territoire catalan. D'après lui, cela est pourtant essentiel dans la construction idéologique d'un peuple : « on défend la cause de notre travail, on proclame l'injustice de nos aspirations à gouverner", "nous avons pris conscience de notre individualité nationale ", "tout cela n'est pas assez pour [...] les fondements d'un peuple » ( CASELLAS, Raimon. Brouillon d'article «La forma física de Catalunya », $3 \mathrm{f}$.. ; BNC, Manuscrits, fonds Raimon Casellas [Bloc 1/4] Ms. 5234/6).

91. - « Les frontières du territoire catalan sont : au nord, les départements français de l'Aude et de l'Ariège, occupés par des groupes de populations parlant des dialectes languedociens, et le Val d'Aran, appartenant politiquement à la Catalogne espagnole et habité par des populations qui parlent un dialecte gascon; à l'ouest et au sud, les provinces espagnoles de Huesca, Saragosse, Teruel, Cuenca, Albacete et Murcie, les habitants desquelles parlent des variétés de la langue castillane ; au sud-ouest, est et nord-est, la Méditerranée ». Il faut y ajouter « les Baléares [...], territoire insulaire de langue catalane ». CASELLAS, Raimon. «Les Terres catalanes ». Catalònia, 13 janvier $1900,2^{\mathrm{e}}$ série, $\mathrm{n}^{\circ} 2$, p. 4-5.

\section{RÉSUMÉS}

En 1901, le parti catalaniste de la Ligue régionaliste arrive en tête des élections municipales de Barcelone. Considérant que la Catalogne est culturellement différente du reste de l'Espagne, il aspire à faire de cette région un État-nation indépendant. Dans ce contexte, on assiste à la définition d'une architecture typiquement catalane, bien distincte de l'architecture espagnole, qui vient appuyer le discours catalaniste. Dès lors, il est pertinent de se demander comment le discours sur l'architecture participe à démontrer que la Catalogne est une région profondément 
différente de l'État espagnol, pouvant légitimement accéder au statut d'État-nation indépendant. L'analyse des écrits produits et conservés entre 1901 et 1905 par le critique d'art, journaliste, collectionneur et écrivain indépendantiste catalan Raimon Casellas (1855-1910) révèle, à travers l'étude de l'architecture, qu'il existerait une identité culturelle catalane, indépendante des frontières politiques. L'architecture catalane, construite en réaction à ses deux pays frontaliers que sont l'Espagne et la France, est tout à fait spécifique. Paradoxalement, ce sont les discours étrangers français, et non espagnols, qui contribuent indirectement à fabriquer une architecture nationale catalane dont le but est de servir le message catalaniste en faveur de l'indépendance de la Catalogne vis-à-vis du royaume d'Espagne.

In 1901, the Catalanist party, the Regionalist League, won the municipal elections in Barcelona. Seeing Catalonia as culturally different from the rest of Spain, it aimed to make the region an independent nation-state. In this context, a definition of typical Catalan architecture, distinct from Spanish architecture, supported the Catalanist discourse. It seems relevant then to wonder how discourse about architecture took part in demonstrating that Catalonia is an entirely different region from Spain, a region which can legitimately claim independent nation-state status. The analysis of the writings of the Catalan and pro-independence art critic, journalist, collector and author Raimon Casellas (1855-1910), preserved for the years between 1901 and 1905, shows, through their architectural studies, that there was a Catalan cultural identity, independent of political borders. Catalan architecture, which emerged in reaction to both of its bordering countries, Spain and France, is specific and genuine. Paradoxically, it was the French discourse and not the Spanish discourse which contributed indirectly to the identification of a Catalan national architecture with an objective of furthering the Catalanist message in favour of the independence of Catalonia.

\section{INDEX}

Keywords : Franco-Spanish border, Catalonia, Catalan national architecture, nationalism, Catalan nationalism, Raimon Casellas, regional architecture, nineteenth-twentieth centuries, modernism, Art Nouveau, nation

Mots-clés : frontière France Espagne, Catalogne, fabrication architecture nationale catalane, nationalisme, catalanisme, Raimon Casellas, architecture régionale, xixe-xxe siècles, Modernisme, nation, civilisation

\section{AUTEUR}

\section{LUCIE ABDUL-LÉVÊQUE}

Diplômée de master 2 d'histoire de l'art, université de Strasbourg ; professeur de français, Barcelone lucie.abd@gmail.com 